\title{
Simply shapely: Relative, not absolute shapes are primed in pop-out search
}

\author{
Stefanie I. Becker
}

Published online: 8 May 2013

(C) The University of Queensland; Crown Copyright 2013

\begin{abstract}
Visual search is typically faster when the target from the previous trial is repeated than when it changes. This priming effect is commonly attributed to a selection bias for the target feature value or against the nontarget feature value that carries over to the next trial. By contrast, according to a relational account, what is primed in visual search is the target-nontarget relationship - namely, the feature that the target has in relation to the features in the nontarget context (e.g., larger, darker, redder) - and switch costs occur only when the target-nontarget relations reverse across trials. Here, the relational account was tested against current feature-based views in three eye movement experiments that used different shape search tasks (e.g., geometrical figures varying in the number of corners). For all tested shapes, reversing the target-nontarget relationships produced switch costs of the same magnitude as directly switching the target and nontarget features across trials ("full-switch"). In particular, changing only the nontargets produced large switch costs, even when the target feature was always repeated across trials. By contrast, no switch costs were observed when both the target and nontarget features changed, such that the coarse target-nontarget relations remained constant across trials. These results support the relational account over feature-based accounts of priming and indicate that a target's shape can be encoded relative to the shapes in the nontarget context.
\end{abstract}

Keywords Visual search · Eye movements - Attention . Shape $\cdot$ Priming of pop-out $\cdot$ Contextual $\cdot$ Relational account

S. I. Becker $(\bowtie)$

School of Psychology, University of Queensland,

Brisbane, Australia

e-mail: s.becker@psy.uq.edu.au
Studies on visual search have shown that the targets and nontargets encountered on the previous trial modulate search on the current trial. For instance, in visual search for a pop-out target that was randomly either red or green, Maljkovic and Nakayama (1994) found that responses were faster when the color of the target from the previous trial was repeated than when the target and nontarget colors switched. Corresponding intertrial effects have been found with respect to other target-defining features, such as shape, size, luminance, and orientation (Becker, 2010a, 2010b; Hillstrom, 2000; Huang, Holcombe, \& Pashler, 2004), as well as in tasks in which search was inefficient (e.g., conjunction search; Becker \& Horstmann, 2009; Geyer, Müller, \& Krummenacher, 2006; Kristjánsson \& Driver, 2008; Kristjánsson, Wang, \& Nakayama, 2002). According to the priming of pop-out hypothesis (Maljkovic \& Nakayama, 1994), attending to the target on a given trial primes or biases attention to select the same feature on subsequent trial(s). The selection bias for the previously selected target feature facilitates search when the target feature is repeated, and impairs target selection when the target and nontarget features reverse relative to the previous trial ("switch costs").

In line with this attentional-biasing hypothesis, several eye movement studies have found that switching the target and nontarget features produces a misguidance effect: When the target and nontarget features from the previous trial switched, one of the inconspicuous nontargets was frequently selected prior to the target. By contrast, repeating the target and nontarget features from the previous trial led to a high proportion of first eye movements to the target (Becker, 2008a, 2008b, 2008c; Becker \& Horstmann, 2009; McPeek, Maljkovic, \& Nakayama, 1999). These results show that priming indeed changes the attentional weights or gains of the target feature (target activation) and/or of the nontarget feature (nontarget inhibition), and does not modulate the speed of target selection (as would be expected according to, e.g., an episodic-retrieval view 
of priming, that repeating the search display merely speeds the retrieval of memory traces without changing attentional gains; Becker, 2008b; Brascamp, Blake, \& Kristjánsson, 2011).

Originally, an attentional bias was thought to operate on the specific target or nontarget feature values (or the corresponding feature maps), and feature-priming effects were supposedly driven by intertrial transfers of feature-specific information (e.g., Lamy, Antebi, Aviani, \& Carmel, 2008; Leonard \& Egeth, 2008; Maljkovic \& Nakayama, 1994). However, recent research has called this view into question.

According to a feature-based account, priming effects should emerge when either the target changes or the nontarget features change, as compared to the previous trial (e.g., Kristjánsson \& Driver, 2008; Lamy et al., 2008; Maljkovic \& Nakayama, 1994). Moreover, as the target and nontarget features are coded independently on separate feature maps, the switch costs arising from changes of the target feature and nontarget feature should be additive (e.g., Lamy et al., 2008). By contrast, Becker (2008a, 2010a) found that both the target and nontarget features can change across trials without incurring any switch costs. For example, if in one set of trials the target is small and presented among medium nontargets, and in the other set of trials the target is large and presented among extra-large nontargets, switching between the sets does not produce switch costs. On the other hand, changing the target size between small and large among consistently medium nontargets incurs significant switch costs that are of the same magnitude as directly switching the target and nontarget features across trials (e.g., medium target among small nontargets, and vice versa; Becker 2008a, 2010a).

These findings have been taken to show that switch costs depend on the coarse target-nontarget relationships: When the target was consistently the smallest item in all displays (always smaller than the nontargets), changing the target and/or nontarget features did not incur switch costs. Switch costs have only been observed when the coarse relationships between the target and nontarget features reversed across trialsthat is, when the target changed from being larger to being smaller than the nontargets, or vice versa.

To explain these results, Becker (2008a) proposed a relational-priming hypothesis. According to this view, the target feature is usually encoded relative to the features of the nontarget context, and attention is subsequently biased toward the relative feature of the target (e.g., its being larger), not its particular feature value (e.g., $5 \mathrm{~cm}$ large). Information about the relative properties of the target (e.g., smaller or larger) is then transferred to the next trial and biases attention to items that have the same feature relationships to the other items as the target had to the nontargets on the previous trial (Becker, 2008a, 2010a). This leads to intertrial switch costs when the target-nontarget relationships reverse across trials (e.g., target smaller/larger than nontargets, or target redder/greener). By contrast, switch costs should be absent when the target has the same relations to the nontargets as on the previous trialregardless of whether the target or nontarget features themselves have repeated or changed.

\section{Evidence for the relational account versus feature-based accounts of priming}

Although previous studies have rejected a feature-based view of priming in size search, it is not yet clear whether these results will generalize to search in other stimulus dimensions. In search for an object with a particular size, a relational search strategy may be preferred to featurespecific search, because the size of objects on the retina is very variable: Depending on its distance from the observer, the same object can be small, medium, or large. By contrast, the relative size of an object (e.g., larger) is largely independent of the distance, and thus could provide more reliable information about the target location. Hence, it is possible that previous results concerning size-priming effects could have been due to top-down search strategies of the observers that are adopted only in size search.

To test whether the results from the size search task would generalize to other stimulus dimensions, priming effects were also examined in search for color and luminance pop-out targets. The results from these experiments were also commensurate with the relational account: For instance, randomly varying the target's luminance between light and medium gray did not incur switch costs when the nontargets were always dark gray. On the other hand, varying the target between light and dark gray among medium-gray nontargets produced significant switch costs (Becker, 2010a). These results are in line with the relational account, as switch costs occurred only when the target-nontarget relationships reversed (from lighter to darker, or vice versa), not when the target was consistently lighter than the nontargets.

However, it could be argued that these findings do not provide decisive evidence for the relational account, for two reasons: First, in all experiments, relationship reversals were realized by changing the target feature value. Hence, it is possible that the observed switch costs were due not to changes in the target-nontarget relationship, but to changes in the target feature. At a minimum, target feature changes could have mitigated effects of relationship reversals, and it is unknown whether and to what extent they each contributed to switch costs.

Second, the results from color and luminance search are still consistent with a feature-divider or feature-grouping account. Of note, in the condition in which the target was always lighter than the nontargets, the possible targets were light and medium gray, and thus were quite similar to one another and could be grouped together. Hence, visual selection could have been based on a broader featural classification that distinguished 
the possible targets from the nontargets (e.g., Hodsoll, Humphreys, \& Braithwaite, 2006; Huang \& Pashler, 2005; Wolfe, 1994). Grouping of the possible target and nontarget features is possible whenever the targets can be separated from the nontargets by drawing a straight line through feature space: In this case, attention can be biased to the broad feature region that contains the possible target features, or biased against the region containing the nontarget features (see, e.g., Hodsoll et al., 2006; Huang \& Pashler, 2005; Wolfe, 1994). Separating the possible target features from the possible nontarget features would not have been possible in the condition in which the target could be lighter or darker than the nontargets, because the medium nontargets were located between the possible target features in feature space. According to a feature-grouping or feature-divider account, switch costs could have been due to the requirement to shift the feature divider discriminating between the target and nontargets on switch trials (e.g., Hodsoll et al., 2006; Huang \& Pashler, 2005; see also Bauer, Jolicœur, \& Cowan, 1995; D'Zmura, 1991).

The feature-divider account has been invalidated by the results of the size search task (Becker, 2010a), which showed that switch costs can be eliminated by presenting a consistently smaller target, even when the intertrial changes required a shift of the feature divider (i.e., the target changed from being a small item among medium nontargets to a large item among superlarge nontargets, requiring a shift of the feature divider; Becker, 2010a). Thus, the results from the size search task clearly showed that switch costs depend on reversals of the target-nontarget relationships, not on the requirement to shift a feature divider.

In sum, the case for the relational account so far mainly rests on the findings obtained in size pop-out search (Becker, 2010a). However, even these results can be regarded as dubious, because relationship reversals were always accompanied by a change in the target feature value. Moreover, as was mentioned above, results from size search may not generalize to other stimulus dimensions because the retinal size of an object strongly depends on the distance. Hence, it is still an open question whether priming effects are indeed relational or feature-specific.

\section{Aims of the present study}

The present study was designed to test the predictions of the relational account with a hitherto untested propertynamely, shape. Shapes were deemed to provide a stronger test of the relational account than other features, because the shape of an object can plausibly be encoded in a feature-specific manner and independent of other, irrelevant shapes in the context. Moreover, it is currently unknown whether early visual processes can encode shape in a context-dependent manner (i.e., relative to other shapes in the context), and whether attention can be biased toward a target's relative shape. Hence, testing the relational account in shape search can potentially shed new light on priming effects as well as preattentive encoding mechanisms.

One complication is that it is impossible to vary the shape of an object without changing any other low-level visual properties (see, e.g., Arguin \& Saumier, 2000). To address this problem, the present study tested priming effects in three experiments that used different shapes, thus ensuring that variations in shape were not confounded with the same low-level visual property (e.g., size). In Experiment 1, the shapes were stars that gradually varied in their numbers of spikes. In Experiment 2, abstract stimuli were used that varied in complexity, and in Experiment 3, geometrical figures such as triangles, squares, and pentagons were tested that varied in their numbers of corners (see Fig. 1).

In all experiments, observers had to make a fast eye movement to the target shape and to discriminate a small item inside the target with a buttonpress $(\mathrm{o} / \mathrm{x}$, associated with a left/right buttonpress). The small response-related items were included to encourage eye movements to the center of the items and to prevent saccadic undershoot
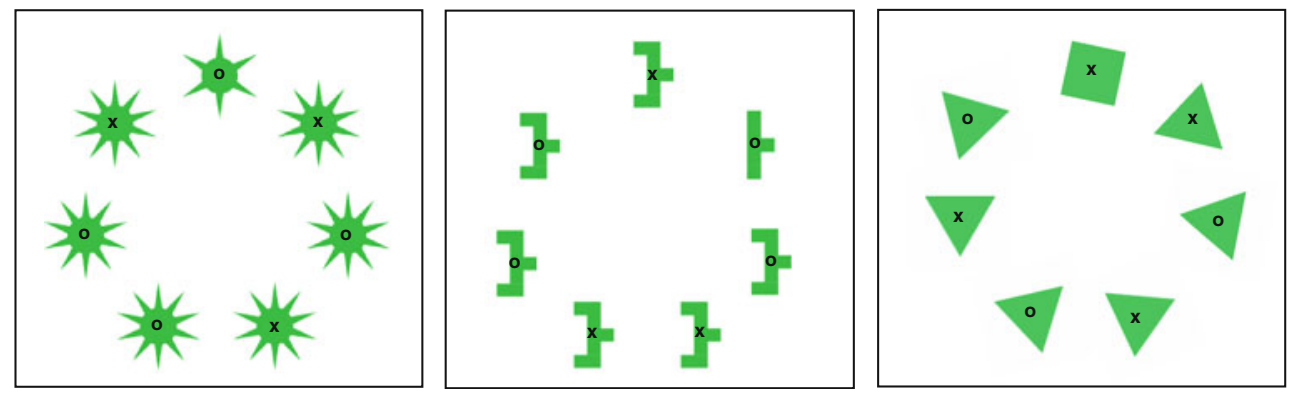

Fig. 1 Example displays for Experiments 1, 2, and 3. Observers had to search for the odd shape and to respond to the item located inside the target shape $(\mathrm{o} / \mathrm{x}=$ right/left buttonpress $)$. The star stimuli used in Experiment 1 varied in their "spikiness" (left panel); the abstract shapes used in Experiment 2 varied in complexity (middle panel), and the geometrical figures used in Experiment 3 varied in their numbers of corners (right panel). Stimuli are not drawn to scale 
(which complicates the assignment of fixations to search stimuli). The main analyses focused on the proportions of first eye movements to the target versus to one of the nontargets, as well as the latencies of these eye movements (i.e., the time needed to initiate the first eye movement to the target). Eye movements were deemed to be a better indicator for covert attention shifts than buttonpress response times (RTs) because a saccade is usually preceded by a covert attention shift to the saccade endpoint (e.g., Deubel \& Schneider, 1996; Hoffman \& Subramaniam, 1995; Kowler, Anderson, Dosher, \& Blaser, 1995) and because eye movements probe visual search performance at an earlier time point, and are thus less affected by later, postselectional processes than are buttonpress RTs (e.g., Becker, 2008a; McPeek et al., 1999).

To test whether the feature-priming effects in search for shape are feature-specific or relational, the target and nontarget features were varied differently in three blocked conditions: In the same-relation condition, the target and nontarget features changed such that the coarse relationship between the target and nontarget features remained the same across the whole block (e.g., target always less spiky; Exp. 1). About half of the same-relation conditions included a partial switch of the target and nontarget features, so that, on switch trials, the target inherited the shape previously associated with the nontargets, and the nontargets adopted a "new" feature not presented on the previous trial, or vice versa (half-switch condition). In the relation-reversal condition, the target and nontarget features changed such that, on switch trials, the relationship between target and nontargets would reverse (e.g., from a spikier target to a less spiky target, or vice versa; Exp. 1). The full-switch condition instantiated the classical condition in which the target and nontarget features were exchanged on switch trials, so that the target inherited the feature formerly associated with the nontargets, and vice versa. The full-switch condition served as a benchmark against which switch costs from the other two conditions could be compared: If switch costs were entirely due to the fact that the target-nontarget relations reversed, the relationreversal condition should yield switch costs of the same magnitude as the full-switch condition.

Table 1 provides an overview of the stimuli and intertrial contingencies tested in Experiments 1-3. As an advance on previous studies, all of the experiments included a condition in which the target always remained constant, and relationship reversals were realized by changing only the nontarget features. Hence, it was possible to test (for the first time) whether relationship reversals would lead to switch costs without simultaneously changing the target feature value. As is shown in Table 1, possible combinations of target feature changes, nontarget feature changes, and relationship changes were chosen such that the relational account could be tested against a target activation account, a nontarget inhibition account, and a feature-divider account. In particular, priming effects were tested when (1) only the target feature changed (Exp. 1A), (2) only the nontarget feature changed (Exps. 1B, 2, and 3), (3) both the target and nontarget features changed, in the manner of a half-switch (i.e., with the previous target feature being the nontarget feature on the current trial, or vice versa; Exps. 2 and $3)$, and (4) the target and nontarget features directly switched (Exps. 1A, 1B, and 2).

According to feature-based accounts, priming could bias visual selection to the previous target shape (target activation account), or it could inhibit selection of the previous nontarget shapes (nontarget inhibition account). In target activation accounts, switch costs should be especially pronounced at changes of the target feature value. According to nontarget inhibition accounts, switch costs should predominantly emerge when the nontarget feature changes across trials. Both target activation and nontarget inhibition accounts predict larger switch costs in a half-switch condition than when the target or nontarget features only change (and adopt a new feature that was not present on the previous trial), because in a half-switch, the target either inherits the previously inhibited feature, or the nontargets inherit a previously activated feature, which actively biases attention against the target feature. Moreover, feature-based accounts typically assume that the target feature is processed independently from the nontarget feature value. Hence, switch costs occurring at changes of the target feature and the nontarget feature should be additive, and together should yield the switch cost observed on trials in which both the target and nontarget features change (e.g., Lamy et al., 2008).

By contrast, according to the relational account, the target feature is not processed independently of the nontarget feature. Instead, attention is biased to the target's relation to the nontargets. For example, when a selected target has more spikes than the nontarget shapes ("spikiest star"), priming would bias selection to the spikiest star on the next trial. This facilitates selection when the target is spikier than the nontargets, and thus again "the spikiest item," but leads to erroneous selection of one of the nontarget shapes when the target is less spiky (and the nontargets are spikier). Importantly, switch trials should impair target selection only when the target's relation to the nontargets changes (e.g., from spikier to less spiky), regardless of whether the target and/or the nontarget feature values change or not.

To focus on switch costs that occurred at an early level of visual selection, the proportions of first eye movements to the target and the latencies of these eye movements were assessed on repetition trials versus switch trials. Other dependent measures, such as the mean RT, error scores, and the number of nontarget fixations prior to target selection are reported for the sake of completeness, and to allow for comparing the present results to those of previous studies, but these measures will not be discussed in detail. 
Table 1 Overview of stimuli, conditions, and predictions

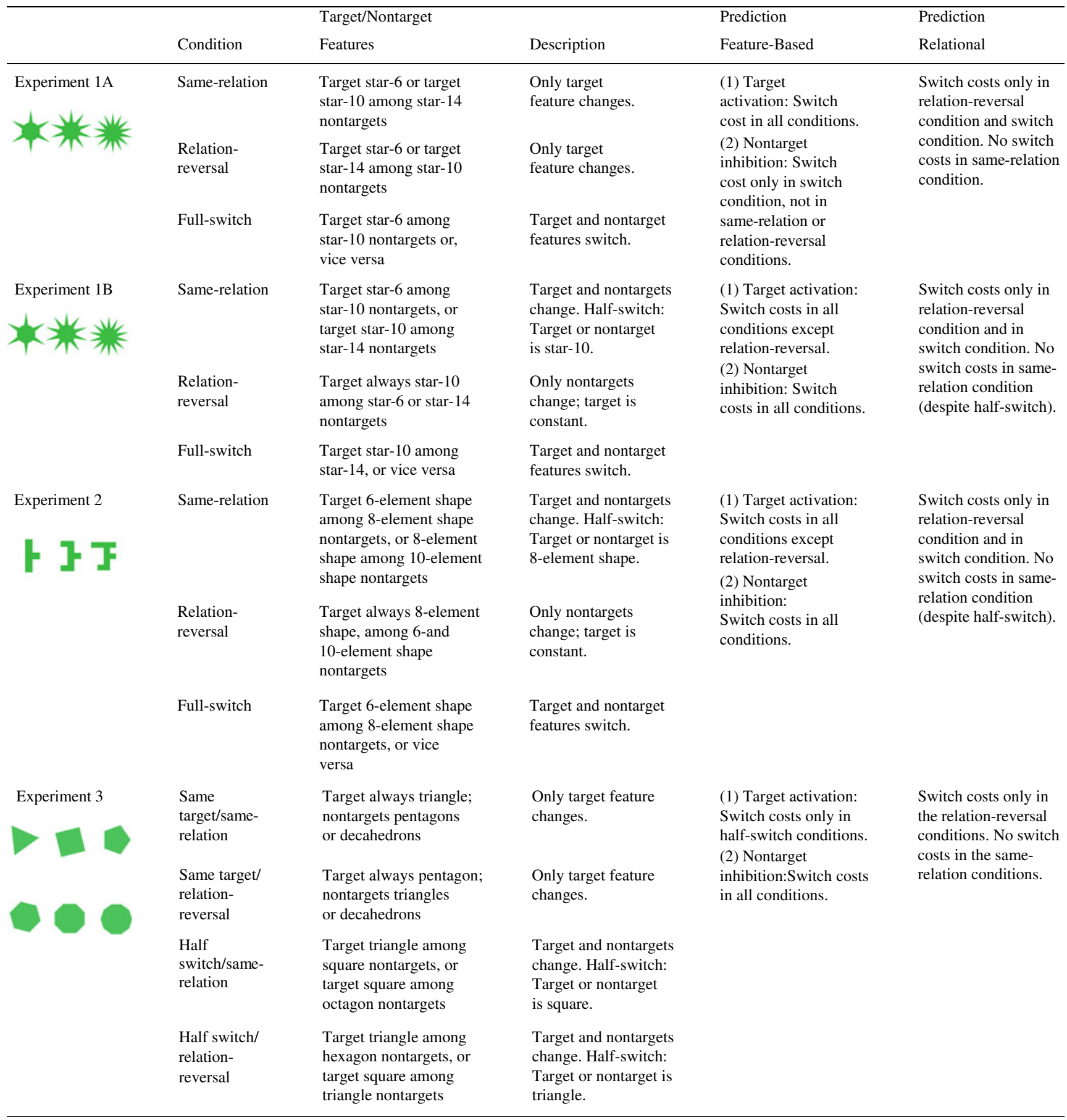

\section{Experiment 1}

In Experiment 1, the target and nontarget stimuli consisted of stars that differed in their numbers of spikes $(6,10$, and 14 spikes). The target-nontarget relationship, or the relative shape of the target, thus consisted in the target being "spikier" or "less spiky" than the nontargets.

Experiment 1 consisted of two experiments that each comprised three blocked conditions. In the same-relation and relation-reversal conditions of Experiment 1A, only the target feature changed, whereas the nontargets always had a constant shape. In the same-relation condition of Experiment 1A, the target randomly varied between a 6-spike star and a 10-spike star, which were both less spiky than the 14-spike nontargets. In the relation-reversal condition, the target could be either a 6-spike star or a 14-spike star, whereas the nontargets were 10spike stars. Thus, on switch trials, the target's relative shape changed from being spikier to being less spiky, or vice versa. 
Switch costs occurring in either of these conditions could then be compared to those from a full-switch condition in which the target and nontarget features directly switched (with the target being a 6-spike star among 10-spike nontarget stars, or vice versa). The conditions of Experiment $1 \mathrm{~A}$ were identical to conditions previously tested in search for color and luminance targets (Becker, 2010a) and were mainly included in order to compare the shape-priming effects to those previous results.

Experiment 1B similarly consisted of three blocked conditions. However, in the relation-reversal condition, the target always had the same feature value, and reversals of the target-nontarget relationship were achieved by varying only the nontarget features (i.e., the target star had 10 spikes and was presented among 6- or 14-spike stars). In the samerelation condition, the target and nontarget features both changed, in the manner of a half-switch: The target was randomly either a 6-spike star among 10 -spike nontarget stars, or a 10-spike target star among 14-spike nontarget stars. Thus, the target was consistently less spiky than the nontargets, but could not be selected by virtue of its feature values and would require adjusting the position of a feature divider on switch trials (because the 10-spike star was a feature of the target on half of all trials, and a feature of the nontargets on the remaining half). The third condition was the full-switch condition, which again served as a control, with the target being either a 10-spike star among 14-spike nontarget stars, or vice versa.

The predictions were as follows: According to the relational account, switch costs are caused by reversals of the targetnontarget relationship. Hence, there should be no switch costs in the same-relation conditions. Plus, switch costs in the relation-reversal condition should be of the same magnitude as in the full-switch condition. A corresponding result pattern would support the relational-priming account and indicate that priming biases visual selection toward the relative shape of the target, and not its absolute shape. According to a target activation account, switch costs should occur in response to changes of the target feature value, and thus should be present in all conditions except the relation-reversal condition of Experiment 1B, in which the target was always constant. A nontarget inhibition view predicts that switch costs should surface when the nontarget features change, which would be the case in all conditions except the samerelation and relation-reversal conditions of Experiment 1A. Feature-grouping or feature-divider accounts predict switch costs in conditions that require a reset of a feature divider, and thus would predict switch costs in all conditions except the same-relation condition of Experiment 1A (see Table 1 for an overview of the predictions).

Method

Participants A group of 12 volunteers from the University of Queensland, Australia, participated in Experiment 1, with the first half participating in Experiment 1A (three males, three females; mean age 27.0 years) and the other half in Experiment 1B (two males, four females; mean age 25.3 years). All participants received $\$ 10$ for their participation, were naive as to the purpose of the experiment, and had normal or corrected-to-normal vision.

Materials An Intel Duo 2 CPU 2.4-GHz computer with a 17-in. FP92E color monitor was used to produce and display the stimuli. Stimuli were presented at a resolution of 1,280 $\times$ 1,024 pixels and a refresh rate of $60 \mathrm{~Hz}$. For eyetracking, a video-based infrared eyetracker with a spatial resolution of 0.1 and a temporal resolution of $500 \mathrm{~Hz}$ was used (EyeLink 1000, SR Research, Ontario, Canada). Participants were seated in a well-lit room with their heads stabilized by the eyetracker's chin rest and forehead support, and they viewed the screen from a distance of $63 \mathrm{~cm}$. For registration of manual responses, a standard USB optical mouse was used. Event scheduling and RT measurements were controlled by the Presentation software (Neurobehavioral Systems, Albany, CA).

Stimuli The response-defining stimuli consisted of seven small black "o" or " $\mathrm{x}$ " characters $\left(0.2^{\circ} \times 0.2^{\circ}\right.$; Arial Black, 12-point) which were located at the center of the search stimuli. The search items were green stars consisting of a central disk $\left(1.2^{\circ}\right)$ and six, ten, or 14 spikes or triangles $\left(0.3^{\circ} \times 0.9^{\circ}\right)$. On each trial, seven green stars were placed equidistant around the outline of an imaginary circle with a diameter of $12^{\circ}$, beginning at the 12-o'clock position. All of the stimuli were presented against a gray background that was equiluminant with the green stars $\left(40.1 \mathrm{~cd} / \mathrm{m}^{2}\right)$. The left panel of Fig. 1 depicts an example of the stimulus displays.

Design Experiments 1A and 1B consisted of three blocked search conditions each. The blocked conditions differed with respect to the possible target and nontarget features, which are described in detail in Table 1. The target position, target type, and response-defining items were chosen randomly on each trial, with the limitation that each display contained an equal number of left and right response-indicative items (excepting the target). The probabilities of repetition and switch trials were $50 \%$. Each block contained 240 trials, and the order of blocks was counterbalanced across participants.

Procedure Each trial started with the presentation of a small black fixation cross and a fixation control: The search display was only presented when the tracking was stable (no blinks), and the gaze was within 50 pixels of the center of the fixation cross for at least $500 \mathrm{~ms}$ (within a time window of 2,000 ms). Otherwise, participants were calibrated anew (randomized nine-point calibration), and the next trial started again with the fixation control. 
Upon presentation of the stimulus display the fixation cross disappeared, and observers were required to make a fast eye movement to the predefined target shape. Observers had to press the right mouse button if the responseindicative item located inside the target square was an "o," and the left mouse button when it was an "x." The stimulus display remained on screen until the manual response and was immediately succeeded by a feedback display. Feedback consisted of the black printed words "right" or "wrong" (Arial, 12-point), which were presented centrally against a gray background and remained on screen for $500 \mathrm{~ms}$. After an intertrial interval of $250 \mathrm{~ms}$, in which a blank gray screen was presented, the next trial started with the presentation of the fixation cross.

Before the experiment, participants were instructed to make a fast and precise eye movement to the target and to respond as quickly as possible without making any mistakes. Before each block, the possible target and nontarget features were described to the participants, either verbally (Exp. 1A), or in written instructions that contained examples of the two possible search displays (Exp. 1B). Participants were encouraged to use the information about the possible target and nontargets. On average, it took $40 \mathrm{~min}$ to complete the experiment.

\section{Results}

Data Eye movements were parsed into saccades, fixations, and blinks using the standard parser configuration of the EyeLink, which classifies an eye movement as a saccade when it exceeds a velocity of $30 \%$ or an acceleration of $8,000^{\circ} / \mathrm{s}^{2}$. Fixations were assigned to the target or a nontarget if the gaze was within 100 pixels $\left(1.0^{\circ}\right)$ of the center of the search item.

In this and all subsequent experiments, trials with RTs below $200 \mathrm{~ms}$ and above 3,000 ms were excluded from all analyses, as were trials in which the eyes had not fixated the target within 3,000 ms after trial onset. Trials with manual errors were similarly excluded from the analysis of RTs and eye movements, to ensure that the results were based on the same trials and could be directly compared. In Experiment $1 \mathrm{~A}, 0.79 \%$ of the data were excluded because of RT outliers, and $1.11 \%$ of the data because of fixation failures. In Experiment 1B, $4.67 \%$ of the data were excluded because of RT outliers, and $1.55 \%$ because of fixation failures.

Data were analyzed with repeated measures analyses of variance (ANOVAs) and two-tailed $t$ tests. For the statistical analyses, Greenhouse-Geisser corrected $p$ values are reported (where appropriate), together with the uncorrected degrees of freedom.

Proportions of first eye movements to the target The mean proportions of first fixations on the target are depicted in Fig. 2.
For a first analysis, two $3 \times 2$ ANOVAs with the variables condition (same-relation vs. relation-reversal vs. full-switch) and priming (repetition vs. switch trial) were computed over the proportions of first target fixations of both Experiments 1A and 1B. The results showed significant main effects of search condition [Exp. 1A, $F(2,10)=32.0, p<.001, \eta^{2}=.86$; Exp. $\left.1 \mathrm{~B}, F(2,10)=6.0, p=.027, \eta^{2}=.55\right]$, priming [Exp. 1A, $F(1,5)=12.2, p=.018, \eta^{2}=.71 ;$ Exp. 1B, $F(1,5)=44.4$, $\left.p=.001, \eta^{2}=.90\right]$, as well as a significant Condition $\times$ Priming interaction [Exp. 1A, $F(2,10)=19.8, p<.001, \eta^{2}=.80$; Exp. $\left.1 \mathrm{~B}, F(2,10)=13.1, p=.012, \eta^{2}=.72\right]$. Pair-wise $t$ tests showed that switch trials did not affect first fixations in the same-relation condition $(t<1)$ but did significantly impair target selection in the relation-reversal condition [Exp. 1A, $t(5)=2.8, p=.04$; Exp. 1B, $t(5)=5.2, p=.003]$ and in the full-switch condition [Exp. 1A, $t(5)=8.1, p<.001$; Exp. 1B, $t(5)=6.7, p=.001]$. A $2 \times 2$ ANOVA comparing priming effects in the relation-reversal and full-switch condition showed no significant differences in the magnitudes of the priming effects [Exp. 1A, $F(1,5)=2.7, p=.16$; Exp. 1B, $F(1,5)=3.7, p=.11]$.

Latencies of first fixations on the target The mean target fixation latencies are depicted in Table 2. For Experiment $1 \mathrm{~A}$, the same $3 \times 2$ ANOVA computed over the mean target fixation latencies showed only a marginally significant main effect of the target condition $\left[F(1,5)=5.4, p=.059, \eta^{2}=.52\right]$, reflecting that target fixation latencies were shorter in the same-relation condition than in the relation-reversal condition $\left[F(1,5)=11.8, p=.018, \eta^{2}=.70\right]$ and the full-switch condition $\left[F(1,5)=6.7, p=.049, \eta^{2}=.57\right]$, whereas they did not differ significantly between the latter two conditions $[F(1,5)=2.9, p=.15]$.

In Experiment 1B, target fixation latencies were significantly affected by priming $\left[F(1,5)=6.9, p=.046, \eta^{2}=.58\right]$, whereas the main effect of block and the interaction failed to reach significance (both $p \mathrm{~s}>.05$ ). As is shown in Table 3, switch trials significantly delayed target selection in the fullswitch condition $[t(5)=3.1, p=.025]$, but not in any of the other conditions $(t \mathrm{~s}<1)$. Thus, interpretation of the data is not complicated by a speed-accuracy trade-off.

\section{Discussion}

The results of the first experiment support the relationalpriming hypothesis: Changing the target in the same-relation conditions did not affect eye movements to the target, presumably because the relative shape of the target did not change (the targets were always less spiky). By contrast, changing the target feature in the relation-reversal conditions did interfere with search and significantly reduced the proportion of first eye movements to the target. Moreover, the switch costs were of the same magnitude in the relation- 
a Exp. 1A: Mean Proportion of $1^{\text {st }}$ Target Fixations

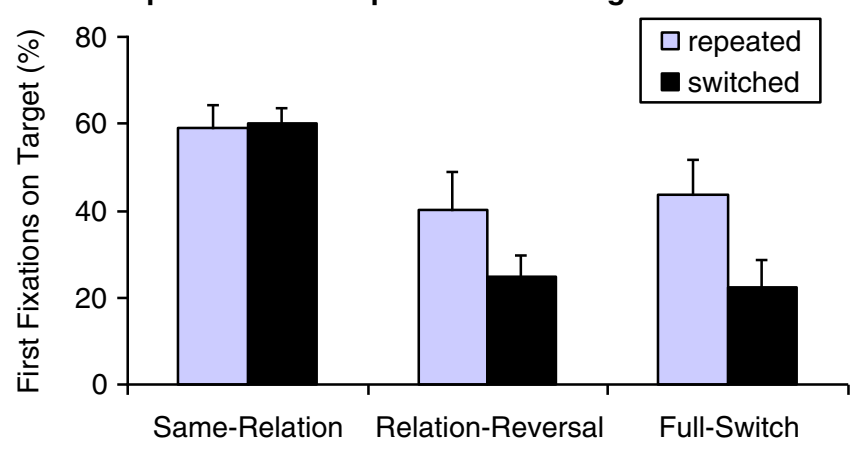

Fig. 2 Mean target selection rates for Experiments 1A and 1B: Proportions of first eye movements to the star-shaped targets in Experiments $1 \mathrm{~A}$ and $1 \mathrm{~B}$, depicted separately for repetition versus switch trials in each of the conditions. Significant switch costs were observed only

reversal and full-switch conditions. These findings support the relational view, that priming biases visual selection to the properties that the target has in relation to the nontargets (e.g., spikier or less spiky).

By contrast, the results are inconsistent with a target activation or nontarget inhibition view of priming. According to a target activation view, we would have expected switch costs in the same-relation condition of Experiment 1A, where the target changed between a 6 - and a 10 -spike star, contrary to

Table 2 Mean target fixation latencies, numbers of nontarget fixations, response times, and errors for Experiment $1 \mathrm{~A}$

\begin{tabular}{llccc}
\hline Dep. Measure & Condition & $\begin{array}{l}\text { Same- } \\
\text { Relation }\end{array}$ & $\begin{array}{c}\text { Relation- } \\
\text { Reversal }\end{array}$ & $\begin{array}{l}\text { Full- } \\
\text { Switch }\end{array}$ \\
\hline Latency $1^{\text {st }}$ Fix. & Repeated & 260 & 275 & 295 \\
& Switched & 258 & 283 & 321 \\
& Priming & -2 & 9 & 26 \\
& & & & \\
No. of Nontarget & Repeated & 0.6 & 1.0 & 0.8 \\
Fix & Switched & 0.5 & 1.4 & 1.2 \\
& Priming & -0.1 & $0.4^{*}$ & $0.4^{* *}$ \\
& Repeated & 845 & 998 & 932 \\
RT (ms) & Switched & 830 & 1,158 & 1,079 \\
& Priming & -15 & $161^{* *}$ & $147^{* *}$ \\
& & & & \\
Error (\%) & Repeated & 9.7 & 7.4 & 6.8 \\
& Switched & 7.3 & 13.7 & 10.0 \\
& Priming & -2.4 & 6.3 & 3.3 \\
\hline
\end{tabular}

The latency of the first target fixation is computed as the time from the onset of the trial to the onset of the saccade directed to the target. The mean number of nontarget fixations was computed from the onset of the trial to the first fixation on the target, with successive fixations on the same stimulus counted as a single fixation. Priming effects were computed as the difference score between switch trials and repeat trials. ${ }^{*} p<.05,{ }^{* *} p<.01$, according to a two-tailed $t$ test. b

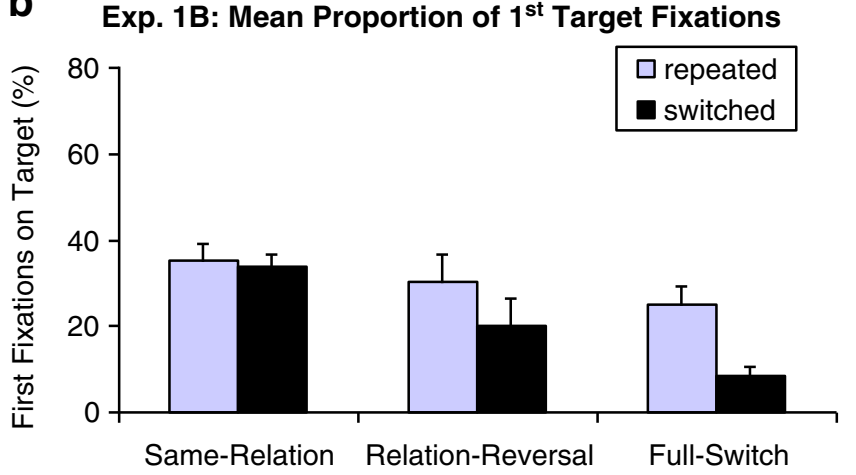

in the relation-reversal condition and the full-switch condition, not in the same-relation condition. Error bars depict the standard errors of the means $(+1 S E M)$

the observations. Moreover, switch costs should have been absent in the relation-reversal condition of Experiment 1B, where the target always had the same shape, also contrary to the results. According to a nontarget inhibition view, switch costs should have been absent in the same-relation and relation-reversal conditions of Experiment 1A, where the nontargets were always repeated (see, e.g., Kristjánsson \& Driver, 2008; Lamy et al., 2008). Moreover, switch costs should have occurred in the same-relation condition of Experiment 1B,

Table 3 Mean target fixation latencies, numbers of nontarget fixations, response times, and errors for Experiment 1B

\begin{tabular}{llccc}
\hline Dep. Measure & Condition & $\begin{array}{l}\text { Same- } \\
\text { Relation }\end{array}$ & $\begin{array}{c}\text { Relation- } \\
\text { Reversal }\end{array}$ & $\begin{array}{l}\text { Full- } \\
\text { Switch }\end{array}$ \\
\hline Latency 1 ${ }^{\text {st }}$ Fix. & Repeated & 282 & 281 & 294 \\
& Switched & 280 & 291 & 338 \\
& Priming & -2 & 10 & $43^{*}$ \\
No. of Nontarget & Repeated & 1.1 & 1.2 & 1.5 \\
Fix & Switched & 1.2 & 1.5 & 2.3 \\
& Priming & 0.1 & $0.3^{*}$ & $0.8^{* *}$ \\
RT (ms) & Repeated & 1,144 & 1,170 & 1,262 \\
& Switched & 1,210 & 1,280 & 1,569 \\
& Priming & 66 & $110^{* *}$ & $307^{* *}$ \\
& & & & \\
Error (\%) & Repeated & 4.0 & 4.6 & 2.9 \\
& Switched & 5.0 & 4.6 & 6.9 \\
& Priming & 1.0 & 0.1 & $4.1^{*}$ \\
\hline
\end{tabular}

The latency of the first target fixation is computed as the time from the onset of the trial to the onset of the saccade directed to the target. The mean number of nontarget fixations was computed from the onset of the trial to the first fixation on the target, with successive fixations on the same stimulus counted as a single fixation. Priming effects were computed as the difference score between switch trials and repeat trials. ${ }^{*} p<.05,{ }^{* *} p<.01$, according to a two-tailed $t$ test. 
where the nontarget features changed. None of these predictions were borne out by the data.

According to a feature-grouping or feature-divider account, switch costs should have occurred in all conditions of Experiment 1B, contrary to the observation that switch costs were absent in the same-relation condition. As switch costs failed to occur when they were predicted, yet occurred when they were not predicted, it is clear that a combined target activation/nontarget inhibition account cannot explain the results, either: Changes in the target shape were neither necessary nor sufficient to cause switch costs, as the target shape could change without incurring such costs (in the samerelation conditions), and keeping the target shape constant did not eliminate switch costs (in the relation-reversal condition; Exp. 1B). The same was true for changes of the nontarget shape: Changing the nontarget shape did not incur switch costs (in the same-relation condition of Exp. 1B), and keeping the nontarget shape constant did not abolish switch costs (in the relation-reversal condition of Exp. 1A). In sum, the results demonstrate that, in search for shape targets, switch costs are independent of changes of the target and/or nontarget features, and depend only on whether or not the target-nontarget relationships reverse across trials.

\section{Experiment 2}

The results of the previous experiment were taken to show that feature-priming effects in shape search are not featurespecific but relational. However, it is not entirely clear whether visual selection was indeed biased to the relative shape of the target; varying the spikiness of the stars admittedly also varied the density of the spikes, so that attention may not have been biased to the relative shapes of the stimuli, but instead to the "sparsest" or "thinnest" stimuli.

Experiment 2 addressed this potential shortcoming by testing a different stimulus set. In Experiment 2, the search stimuli consisted of abstract shapes that varied in complexity. The simplest shape consisted of six elements, and the two more complex stimuli were construed by adding either two or four elements to this shape (see Fig. 1).

The same conditions as used in Experiment 1B were implemented in Experiment 2: That is, in the same-relation condition, the target was either a six-element shape among eight-element nontarget shapes or an eight-element shape among ten-element nontarget shapes. Thus, the target was always less complex than the nontargets, but it could not be selected in terms of its possible (grouped) feature values, as the eight-element shape was equally probable to be the target or the nontarget shape.

In the relation-reversal condition, the target always had the intermediate, eight-element shape, and the nontargets varied randomly between six- and ten-element shapes. Thus, on switch trials, the target remained the same, but its relationship to the nontargets changed from being more complex to less complex, or vice versa. Target selection rates in this condition were compared to those in a fullswitch condition, in which the target was a six-element shape among eight-element shapes, or vice versa. All other aspects of the experiment were identical to those of Experiment 1B.

\section{Method}

Participants A group of 12 new participants took part in Experiment 2 (five males, seven females; mean age 25.7). All of the participants were paid $\$ 10$ for their participation, were naïve as to the purpose of the experiment, and had normal or corrected-to-normal vision.

Stimuli, design, and procedure The experiment was identical to the previous experiment, with the following exceptions: The stimuli were abstract figures that consisted of different numbers of simple square elements $\left(0.3^{\circ} \times 0.3^{\circ}\right)$, with the simplest stimulus consisting of six elements. Stimuli of intermediate and high complexity were generated by adding two and four square elements to the simple shape, respectively (see Fig. 1, middle panel). All of the stimuli were $1.8^{\circ}$ high and $1.5^{\circ}$ wide, at maximum.

As in Experiment 1, the observers' task was to make a fast eye movement to the predefined target shape and to respond to the item located inside the target. The samerelation, relation-reversal, and full-switch condition each comprised 220 trials, with the order of blocks being counterbalanced across participants.

\section{Results}

Data As in Experiment 1, trials with RTs below $200 \mathrm{~ms}$ or above 3,000 ms were excluded, as were trials in which the eyes had not fixated the target within $3,000 \mathrm{~ms}$ from the onset of the trial. A total of $1.81 \%$ of all data were lost because of RT outliers, and a further $2.18 \%$ because of failures to select the target within $3,000 \mathrm{~ms}$.

Proportion of first eye movements to the target The mean proportions of first fixations on the target are depicted in Fig. 3 . A $3 \times 2$ ANOVA with the variables condition (samerelation vs. relation-reversal vs. full-switch) and priming (repetition vs. change), computed over the proportions of first eye movements to the target, showed significant main effects of condition $\left[F(2,22)=6.4, p=.017, \eta^{2}=.37\right]$, priming $[F(1,11)=$ 45.3, $\left.p<.001, \eta^{2}=.81\right]$, and a significant Condition $\times$ Priming interaction $\left[F(2,22)=14.9, p<.001, \eta^{2}=.58\right]$. Switch trials significantly impaired target selection in the relation-reversal 
Exp. 2: Mean Proportion of $1^{\text {st }}$ Target Fixations

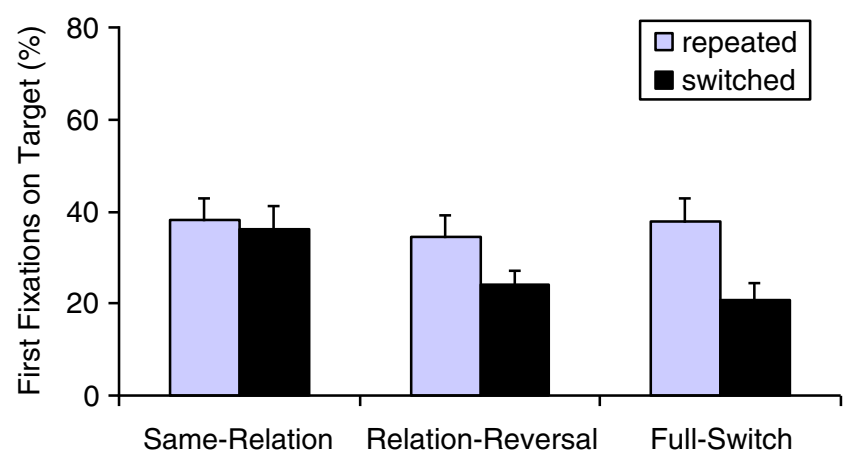

Fig. 3 Mean proportions of first target fixations in Experiment 2, in which the target differed in complexity from the nontarget shapes. The results are depicted separately for repetition versus switch trials in each of the conditions. Significant switch costs were observed in the relation-reversal condition and the full-switch condition, but not in the same-relation condition. Error bars depict the standard errors of the means (+1 SEM)

condition $[t(11)=3.8, p=.003]$ and the full-switch condition $[t(11)=7.5, p<.001]$, but not in the same-relation condition $[t(11)=1.6, p=.14]$. A $2 \times 2$ ANOVA showed no significant differences between the switch costs in the full-switch condition and those in the relation-reversal condition $[F(1,11)=1.8$, $p=.20]$.

Latencies of first fixations on the target The same ANOVA computed over the mean target fixation latencies showed significant main effects of search condition $[F(2,22)=10.3$, $\left.p=.002, \eta^{2}=.48\right]$ and priming $[F(1,11)=22.5, p=.001$, $\left.\eta^{2}=.67\right]$, as well as an interaction between the two variables $\left[F(2,22)=8.3, p=.010, \eta^{2}=.43\right]$. Pair-wise $t$ tests revealed that switch trials significantly delayed eye movements to the target in the relation-reversal condition $[t(11)=4.8, p=.001]$ and the full-switch condition $[t(11)=3.3, p=.008]$, but not in the same-relation condition $(t<1)$. A $2 \times 2$ ANOVA showed that switch costs in the target fixation latencies were larger in the relation-reversal condition than in the full-switch condition $\left[F(1,11)=5.3, p=.042, \eta^{2}=.33\right.$; see Table 4$]$.

\section{Discussion}

The results from Experiment 2 were clear: Intertrial switch costs were due to reversals of the target-nontarget relationships rather than to changes of the target or nontarget feature. This was reflected in the facts (1) that switch costs were absent when the target was consistently less complex than the nontargets, despite the fact that the target and nontarget features changed in the form of a half-switch (same-relation condition), and (2) that changing only the nontargets across trials led to significant switch costs in the relation-reversal condition, which were of a magnitude similar to those in the full-switch condition. These results cannot be explained by a target activation or nontarget
Table 4 Mean target fixation latencies, numbers of nontarget fixations, response times, and errors for Experiment 2

\begin{tabular}{lllll}
\hline Dep. Measure & Condition & $\begin{array}{l}\text { Same- } \\
\text { Relation }\end{array}$ & $\begin{array}{l}\text { Relation- } \\
\text { Reversal }\end{array}$ & $\begin{array}{l}\text { Full- } \\
\text { Switch }\end{array}$ \\
\hline Latency 1st Fix. & Repeated & 289 & 288 & 307 \\
& Switched & 290 & 326 & 335 \\
& Priming & 1 & $38^{* *}$ & $28^{* *}$ \\
No. of Nontarget Fix & Repeated & 1.2 & 1.2 & 1.1 \\
& Switched & 1.2 & 1.5 & 1.6 \\
& Priming & $<0.1$ & $0.3^{* *}$ & $0.5^{* *}$ \\
& & & & \\
RT (ms) & Repeated & 1,044 & 1,041 & 1,070 \\
& Switched & 1,054 & 1,157 & 1,232 \\
& Priming & 9 & $116^{* *}$ & $161^{* *}$ \\
Error (\%) & Repeated & 3.9 & 3.2 & 4.7 \\
& Switched & 4.7 & 3.7 & 6.8 \\
& Priming & 0.8 & 0.6 & $2.1^{*}$ \\
\hline
\end{tabular}

The latency of the first target fixation is computed as the time from the onset of the trial to the onset of the saccade directed to the target. The mean number of nontarget fixations was computed from the onset of the trial to the first fixation on the target, with successive fixations on the same stimulus counted as a single fixation. Priming effects were computed as the difference score between switch trials and repeat trials. ${ }^{*} p<.05,{ }^{* *} p<.01$, according to a two-tailed $t$ test.

inhibition view of priming or by grouping (see Table 1), but instead support the relational account.

In the relation-reversal condition, the switch costs in the proportions of first target fixations were numerically smaller (10\% more nontarget fixations on switch trials) than in the full-switch condition (17\% more nontarget fixations on switch trials). However, these differences cannot be cited in support of a target activation account, as they were not significant. Moreover, a target activation account would appear inconsistent with the absence of switch costs in the same-relation condition, in which both the target and nontargets varied. Taken together, the results indicate that visual selection was not biased to the previous targets' particular shape, but to the previous targets' relative shape, in line with the relational account.

\section{Experiment 3}

It could be argued that interpretation of the results of Experiments 1 and 2 is still problematic, on two counts: First, it is not certain that visual selection was indeed based on the relative shape of the target, because the surface area of the stimuli covaried with differences in shape. Hence, it is possible that attention was biased toward the relative surface area of the target, not its relative shape. A second potential problem is that different types of intertrial changes were 
applied to the same-relation and relation-reversal conditions of Experiments 1B and 2, because a half-switch (in the samerelation condition) was always compared to a mere change in the nontarget features (in the relation-reversal condition).

These asymmetrical conditions were chosen because they allowed for testing the respective theories in the most efficient manner: As the feature-based accounts make three different sets of predictions, pitting a half-switch (samerelation) condition against a condition in which the target shape never changed (relation-reversal) was the most efficient way to test the relational account against target activation, nontarget inhibition, and feature-grouping accounts. However, the design did not permit a full examination of the effects of changing only the nontargets versus both the target and nontargets (in the manner of a half-switch) when the target-nontarget relations remained constant versus reversed.

Experiment 3 addressed these potential shortcomings, by testing familiar geometrical shapes (e.g., triangles, squares, or pentagons) that all covered the same surface area and systematically varied in their numbers of corners (or sides). To ensure that attention was biased toward the shape (or the shape differences) of the target, not local feature differences (e.g., the upward-pointing sharp edge of a triangle vs. the flat surface of a square), the stimuli were all randomly rotated (see the right panel of Fig. 1 for an example).

In addition, Experiment 3 was based on a fully crossed $2 \times 2$ design that permitted testing the effects of relationship reversals (same vs. reversed-relation) when the target feature always remained constant (target-constant condition) versus when both target and nontarget features changed (half-switch condition). In the target-constant/same-relation condition, the target was always a triangle and the nontargets randomly were either pentagons or decahedrons, so that the target consistently had fewer corners than the nontargets. In the target-constant/relation-reversal condition, the target was always a pentagon, and the nontargets were, randomly, either triangles or decahedrons, so that the target-nontarget relationships reversed on switch trials (i.e., from a fewer-cornered to a more-cornered target, or vice versa).

In the half-switch conditions, the target and nontargets could both change across trials, and when they did, the target inherited the shape formerly associated with the nontargets, or vice versa. In the half-switch/same-relation condition, the target consistently had fewer corners than the nontargets (i.e., a triangle target among square nontargets, or a square target among octagon nontargets). In the halfswitch/relation-reversal condition, the target could have either fewer or more corners than the nontargets, so that the target-nontarget relationships reversed on switch trials (i.e., square target among triangle nontargets, or a triangle target among hexagon nontargets; see Table 1).
According to a feature-based account, switch costs should occur in all conditions, if they are caused by changes in the nontarget feature (nontarget inhibition view), or, alternatively, should occur only in the half-switch condition, if they are caused by changes in the target feature (target activation view) or by shifts in the position of a feature divider (feature-grouping account). According to the relational account, switch costs should occur in the relationreversal conditions, in which the target changes between a fewer-cornered item and a more-cornered item, but not in any of the same-relation conditions.

\section{Method}

Participants A group of 12 new participants from the University of Queensland (five males, seven females; mean age 21.4 years) took part in the experiment.

Stimuli, design, and procedure The geometrical shapes used in Experiment 3 were triangles, squares, pentagons, hexagons, octagons, and decahedrons, and thus differed systematically in their numbers of corners. All stimuli had the same surface area $\left(1.69 \mathrm{~cm}^{2}\right)$. To ensure that the target could not be successfully singled out by means of a local feature (e.g., an upward-pointing sharp edge, in the case of a triangle), the stimuli were randomly rotated at their individual positions $\left(0^{\circ}-360^{\circ}\right.$, in steps of $\left.1^{\circ}\right)$.

The experiment consisted of $2 \times 2$ blocked search conditions: In one condition, the target feature always remained constant (target-constant condition), whereas in the other condition, both target and nontarget features changed across trials, in the fashion of a half-switch. Both conditions comprised two blocked conditions: a same-relation condition, in which the target-nontarget relationship was kept constant, and a relation-reversal condition, in which the target-nontarget relationship reversed on switch trials. The stimuli used in each condition are listed in Table 1. Participants completed 150 trials in each of the four blocked conditions, and the order of the blocks was counterbalanced across participants using a Latin square procedure.

Results

Data Excluding all trials with RTs below $200 \mathrm{~ms}$ or above $3,000 \mathrm{~ms}$ resulted in a loss of $1.72 \%$ of all data. Excluding trials in which the eyes had not fixated the target within $3,000 \mathrm{~ms}$ from the onset of trial resulted in a further loss of $0.68 \%$ of the data.

Proportion of first eye movements to the target The mean proportions of first fixations on the target are depicted in Fig. 4. Data were analyzed with a $2 \times 2 \times 2$ ANOVA comprising the variables target change (target feature 
Fig. 4 Mean proportions of first target fixations in Experiment 3, depicted separately for the targetconstant condition and the halfswitch condition, as well as separately for repetition and switch trials in each of the conditions. Significant switch costs were observed in the relation-reversal conditions, but not in the same-relation conditions. Error bars depict the standard errors of the means $(+1$ SEM)

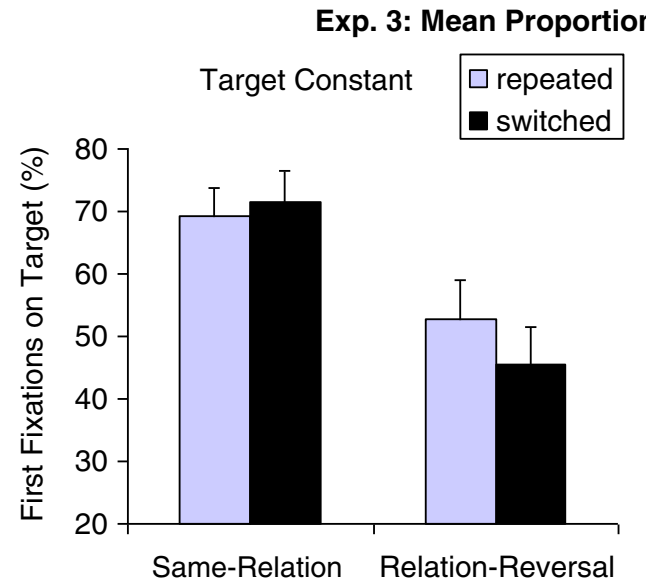

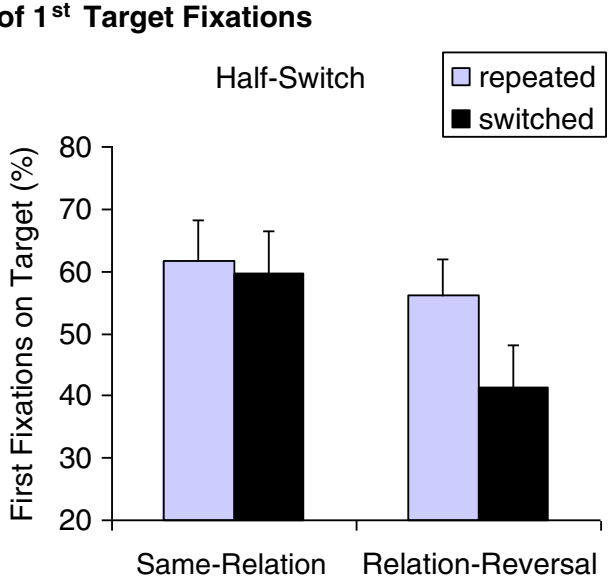

constant vs. half-switch), relation change (same-relation vs. relation-reversal), and priming (repetition vs. switch trial). The results showed significant main effects of relation change $\left[F(1,11)=11.6, p=.006, \eta^{2}=.51\right]$ and priming $\left[F(1,11)=15.0, p=.003, \eta^{2}=.58\right]$, and a significant twoway interaction between the two variables $[F(1,11)=34.6$, $p<.001, \eta^{2}=.76$; all other $\left.p \mathrm{~s}>.05\right]$. Follow-up two-tailed $t$ tests revealed that target selection was significantly impaired on switch trials in the relation-reversal conditions in both the half-switch condition $[t(11)=4.2, p=.002]$ and the targetconstant condition $[t(11)=3.9, p=.003]$. By contrast, switch trials did not impair target selection in the samerelation conditions $(p \mathrm{~s}>.05)$. A $2 \times 2$ ANOVA showed that switch costs in the relation-reversal conditions did not differ between the target feature constant and the half-switch conditions $[F(1,11)=3.2, p=.10]$.

Latencies of first fixations on the target The same $2 \times 2 \times 2$ ANOVA computed over the target fixation latencies showed a significant main effect of target change $[F(1,11)=9.7, p=.010$, $\left.\eta^{2}=.47\right]$, with saccades being elicited earlier in the targetconstant than in the half-switch condition (see Table 5). Moreover, the main effect of relation change was significant $\left[F(1,11)=8.8, p=.013, \eta^{2}=.45\right]$ and interacted significantly with priming $\left[F(1,11)=11.7, p=.006, \eta^{2}=.52\right.$; all other $p \mathrm{~s}>$.05]. Paired $t$ tests showed that saccades to the target were significantly delayed on switch trials in the targetconstant/relation-reversal condition $[t(11)=2.3, p=.045$; all other $p \mathrm{~s}>.05]$.

\section{Discussion}

In Experiment 3, geometrical shapes were used to test effects of relation-reversals when the target feature always remained constant versus when both the target and nontarget features changed in the fashion of a half-switch. The results showed that reversing the target-nontarget relationships significantly impaired visual selection, regardless of whether only the nontarget features varied or both the target and nontarget features changed across trials. The shapes used in Experiment 3 all had the same surface area, ruling out that the results could be attributed to a visual selection bias for the relative size or surface area of the stimuli. Moreover, the stimuli were all rotated, preventing successful selection of the target by virtue of local features that were specific to a certain orientation of the stimuli (e.g., the flat vs. pointed surfaces of squares vs. triangles, respectively). Because of this, the results of Experiment 3 support the relational view,

Table 5 Mean target fixation latencies, numbers of nontarget fixations, response times, and errors for Experiment 3

\begin{tabular}{|c|c|c|c|c|c|}
\hline \multirow[t]{2}{*}{ Dep. Measure } & \multirow[t]{2}{*}{ Condition } & \multicolumn{2}{|c|}{ Target Constant } & \multicolumn{2}{|c|}{ Half-Switch } \\
\hline & & $\begin{array}{l}\text { Same- } \\
\text { Relation }\end{array}$ & $\begin{array}{l}\text { Relation- } \\
\text { Reversal }\end{array}$ & $\begin{array}{l}\text { Same- } \\
\text { Relation }\end{array}$ & $\begin{array}{l}\text { Relation- } \\
\text { Reversal }\end{array}$ \\
\hline \multirow[t]{3}{*}{ Latency 1st Fix. } & Repeated & 273 & 335 & 361 & 414 \\
\hline & Switched & 273 & 354 & 366 & 433 \\
\hline & Priming & $<1$ & $19^{*}$ & 4 & 19 \\
\hline \multirow{3}{*}{$\begin{array}{l}\text { No. of Nontarget } \\
\text { Fix }\end{array}$} & Repeated & 0.3 & 0.7 & 0.6 & 0.6 \\
\hline & Switched & 0.3 & 0.8 & 0.6 & 0.9 \\
\hline & Priming & $<0.1$ & $0.2^{* *}$ & $<0.1$ & $0.3^{* *}$ \\
\hline \multirow[t]{3}{*}{ RT (ms) } & Repeated & 805 & 1,032 & 1,108 & 1,156 \\
\hline & Switched & 808 & 1,103 & 1,179 & 1,310 \\
\hline & Priming & 3 & $71^{* *}$ & $71^{* *}$ & $154^{* *}$ \\
\hline \multirow[t]{3}{*}{ Error (\%) } & Repeated & 4.0 & 3.6 & 3.8 & 3.8 \\
\hline & Switched & 3.8 & 4.0 & 4.4 & 6.7 \\
\hline & Priming & -0.2 & 0.4 & 0.6 & $2.9^{*}$ \\
\hline
\end{tabular}

The latency of the first target fixation is computed as the time from the onset of the trial to the onset of the saccade directed to the target. The mean number of nontarget fixations was computed from the onset of the trial to the first fixation on the target, with successive fixations on the same stimulus counted as a single fixation. Priming effects were computed as the difference score between switch trials and repeat trials. ${ }^{*} p<.05,{ }^{* *} p<.01$, according to a two-tailed $t$ test. 
that priming effects depend more on the relative shape of the target than on the shapes of either the target or the nontargets.

Eye movements to the target were elicited earlier in the target-constant condition than in the half-switch condition, in which the target and nontarget features varied randomly. This indicates that certainty about the target feature and/or perpetual repetitions of the target feature value may aid target selection. However, this result cannot be taken to support a target activation view, as intertrial switch costs were not significantly attenuated in the target-constant conditions. Although switch costs were numerically smaller in the target-constant than in the half-switch condition, the target fixation latencies showed the opposite results, with significant switch costs in the target-constant but not in the half-switch condition. Hence, with respect to the possible effects of target constancy, the only conclusion that we can safely draw is that target selection commenced earlier in the target-constant condition, without target constancy notably affecting intertrial switch costs or the overall probability of selecting the target as the first item.

\section{General discussion}

The results of the present study were clear: In line with the relational account, feature-priming effects did not depend on whether the target or nontarget features changed across trials, but on whether the relation between the target and nontarget shapes reversed or remained constant, as compared to the previous trial.

These results extend on previous research in several ways: Previous studies testing priming in color and luminance search used the same design as the present Experiment 1A (Becker, 2008a, 2010a). The feature-priming effects observed in the relation-reversal condition and the absence of switch costs in the same-relation condition were still consistent with featuregrouping accounts that assume that switch costs are caused by the need to shift a feature divider that discriminates the target from the nontargets (e.g., Hodsoll et al., 2006; Huang \& Pashler, 2005; see also Wolfe, 1994).

Previous results from size search tasks had already invalidated this explanation, as switch costs were absent in same-relation conditions that required a shift in the feature divider (Becker, 2010a). The present study extends these findings to shape search, by showing that conditions that would require a shift in the feature divider (half-switch/same-relation conditions) do not automatically produce switch costs. This supports the relational account over a feature-grouping account or a feature-divider account.

The results of the present study also refute the classical view of feature priming as being caused by a carry-over effect of target activation and/or nontarget inhibition, on two counts: first, by showing that these accounts do not correctly predict the occurrence of intertrial switch costs in the tested intertrial transitions (see Table 1; see also Becker, 2008a). Second, the results undermine the central assumption of the classical view that target and nontarget features are processed independently of one another (e.g., Lamy et al., 2008; Maljkovic \& Nakayama, 1994), by showing that shapes are encoded relative to the nontarget shapes (in feature priming; e.g., as being spikier, more complex, or more cornered). Importantly, contextdependent encoding was demonstrated in shape search in the present study, providing the first evidence that early processes that guide visual attention can encode shapes in a context-dependent manner.

Naturally, the shapes used in Experiments 1-3 also varied in at least one, sometimes two, other low-level properties, as the shape of a stimulus cannot be changed while keeping all other attributes constant. Note, however, that the shapes did not covary with the same low-level property in all experiments, and yet the results were very similar across all experiments. This result would seem to support the view that the target's shape was encoded in a context-dependent manner (although it cannot be ruled out that other low-level properties were used as a substitute to find the target, and that all of these were encoded relationally).

We can also ask why early perceptual processes would encode feature relationships rather than the absolute feature values of the target and nontargets in search for a particular shape. This question cannot be answered with certainty. In a complex and unpredictable environment, a relational encoding strategy could be more efficient than a feature-specific encoding strategy, because it does not require detailed foreknowledge of the target and can be flexibly applied to a large number of different situations and stimuli ("one code to rule them all") ${ }^{1}$. However, this argument is certainly speculative and warrants further research.

\section{Explaining evidence for target activation and nontarget} inhibition

The results from the present study may appear inconsistent with previous studies reporting that changing the target and nontarget features have dissociable effects and impair search independently of each other (e.g., Kristjánsson \& Driver, 2008; Lamy et al., 2008; Maljkovic \& Nakayama, 1994). However, none of the previous studies controlled for reversals of the target-nontarget relationships, and in fact, the reported findings seem perfectly consistent with the relational account.

Maljkovic and Nakayama (1994) were the first to examine the contributions of target activation and nontarget inhibition to priming effects. In a color search task, the target

\footnotetext{
${ }^{1}$ I would like to thank Paul Jackson for suggesting this phrase.
} 
could have one out of four possible colors (red, green, blue, and yellow), and priming effects were compared when only the target color changed versus when only the nontarget color changed. The results showed larger switch costs when the target color changed than when the nontarget color changed, leading Maljkovic and Nakayama to conclude that target activation played a larger role in priming than did nontarget inhibition. Lamy et al. (2008) reinvestigated these effects with the same colors, but assigned the colors randomly to the target and nontargets, which resulted in seven different types of intertrial transitions. The results showed that changing the target color did not interact with changes of the nontarget color. Moreover, changing the target color led to dissociable and larger switch costs than did changing only the nontarget color. Correspondingly, Lamy et al. concluded that target activation had a larger impact on priming effects than did nontarget inhibition.

However, with the configuration of colors used in the experiments, the relational account would have made the same predictions as a combined target activation/nontarget inhibition account. Three of the colors used in the studies (red, yellow, and green) were aligned in CIE color space, with yellow being positioned directly between red and green. Hence, changing the target and/or nontarget colors between these three colors would lead either to a repetition of the previous target-nontarget relationship or to a direct reversal (e.g., redder to greener target). The upshot is that the probability of reversing the target-nontarget relationships (e.g., from redder to greener) was much higher when the target feature changed than when only nontarget features changed. Note that the target has to change from an intermediate color (yellow among red) to a more extreme color (e.g., green among red), or vice versa, to realize a change of the target color without reversing the target-nontarget relationship. However, the only intermediate color was yellow, and the probability of the target being yellow was quite low (25\%). Hence, the majority of target change trials included reversals of the target-nontarget relationships. For a change of the nontargets to lead to relationship reversals, the target would also have to be yellow, with the nontargets switching from red to green or vice versa. Because the probability of the target being yellow was lower (25\%) than that of the target having an extreme feature value (e.g., either red or green; $50 \%$ ), the majority of nontarget change trials did not include relationship reversals. Hence, the relational account would predict larger switch costs in a target change condition than in a nontarget change condition, because target changes are associated with a higher probability of relationship reversals than are nontarget changes (Becker, 2008a, 2010a).

A limitation of the relational account is that not all featural changes can be clearly classified as preserving the previous feature relationship or reversing it (e.g., transitions from blue to red cannot be classified as relation-reversals when the nontargets are yellow or green, because the change in direction does not equal $0^{\circ}$ or $180^{\circ}$ ). Despite these uncertainties, the relational account seems consistent with previous studies reporting, for example, larger switch costs on full-switch than on half-switch trials, and larger switch costs on half-switch than on target change or nontarget change trials (e.g., Eimer, Kiss, \& Cheung, 2010; Kristjánsson \& Driver, 2008; Lamy et al., 2008), because, in the most commonly used stimulus sets, these different types of intertrial changes are confounded with progressively lower probabilities for relationship reversals. ${ }^{2}$

The priming effect: Bottom-up or top-down contingent?

Feature relationships have also been found to modulate attention when the target and nontarget features were kept constant across a block of trials. Using a spatial-cueing task, Becker, Folk, and Remington (2013) showed that, in a search for an orange target among three yellow-orange nontargets, the target-nontarget relationship (redder) and not the feature value of the target (orange) determined which cues were able to attract attention. A singleton cue embedded in a context of three other cues captured attention only when the cue-cue-context relationships matched the target-nontarget relationships, regardless of the specific colors of the cues (e.g., a yellow-orange cue among yellow other cues captured attention, as did a red cue among orange cues, but an orange cue among red other cues did not capture attention; Becker, Folk, \& Remington, 2010, 2013). These and other results were commonly taken to show that visual selection may be generally context-dependent-in "top-down" contingent capture as well as "bottom-up" feature priming (e.g., Becker 2010a; Becker et al., 2010).

Naturally, when the target and nontarget features remain constant over a block of trials, it cannot be determined whether relational effects are due to automatic feature priming or to top-down tuning to a known feature value (e.g., Kristjánsson et al., 2002). A much debated question is whether feature priming effects are top-down-controlled or stimulus-driven, with some researchers arguing that feature-priming effects are part of the top-down selection mechanism (e.g., Becker, 2008a, 2010a; Folk \& Remington, 2008; Wolfe, Butcher,

\footnotetext{
${ }^{2}$ The relational account also seems consistent with previous neurophysiological studies in which priming effects have been examined with functional imaging or single-cell recordings (e.g., Bichot \& Schall, 2002; Kristjánsson, Vuilleumier, Schwartz, Macaluso, \& Driver, 2007): As these studies did not systematically vary the feature relationships, it is unclear whether priming modulated the response gain of neurons responding to a specific feature value or to a feature relationship (e.g., in the fashion of $\mathrm{L}-\mathrm{M}$ opponent color cells; see Becker, 2013, for a detailed discussion).
} 
Lee, \& Hyle, 2003), and others arguing that feature-priming effects are mediated by a separate, bottom-up-controlled attentional system (e.g., Awh, Belopolsky, \& Theeuwes, 2012). The debate is perhaps mainly due to the fact that priming effects can be modulated by top-down-controlled processes without being completely cognitively penetrable: Priming effects are contingent on top-down task demands, in that (1) they are stronger for task-relevant than for task-irrelevant features of the target (e.g., Becker, 2008a, 2008b, 2008c, 2010a, 2010b; Huang et al., 2004; Kristjánsson, 2006); (2) they are eliminated by a switch in the search task (e.g., search for a shape vs. a color target; Fecteau, 2007); and (3) priming effects can be attenuated by instructions to prepare for the upcoming target feature (e.g., Hillstrom, 2000; Leonard \& Egeth, 2008; Maljkovic \& Nakayama, 1994). At the same time, priming effects cannot be completely eliminated by preparing for the upcoming target, or by changing the target in a regularly alternating sequence (e.g., AABBAABB ...), suggesting that they are in part top-down impenetrable (e.g., Becker, 2008b; Hillstrom, 2000).

As a solution to the debate, Folk and Remington (2008) suggested that the top-down task demands determine whether or not priming effects can modulate selection. Specifically, they proposed that feature-priming effects can only come into play when the target feature is uncertain-namely, when the target can have more than a single possible feature value. According to their view, the requirement to attend to multiple possible target features requires distributing top-down attentional gains over all feature maps that correspond to possible target features. With these distributed top-down weight settings in place, priming can modulate selection by enhancing the gain of the last-selected target feature. However, the last-selected item cannot exert an effect when the target feature is certain, and the corresponding feature map is nulled by top-down-controlled processes. In line with this hypothesis, Folk and Remington showed that priming effects can only modulate capture by irrelevant precues when the target could be randomly either red or green. When one of the targets was changed to a no-go target, priming effects were observed only for the go target (which led to stronger capture by the same-colored cue on the next trial), not for the no-go target (Folk \& Remington, 2008). These results support the view that conditions that permit biasing attention to a single target feature can eliminate priming effects for all features except the task-relevant feature.

The results of the present study, however, do not support the view that knowledge about the target feature value abolishes priming effects: Reversing the target-nontarget relationship led to significant switch costs, even when the target feature value was always constant and participants knew the relevant feature in advance (Exps. 1B, 2, and 3). Switch costs were eliminated only in the same-relation conditions, in which the target always had a constant relative shape (including when it could have two different feature values). These results suggest that if priming effects are top-down contingent, they are contingent on knowledge about the relative, not the absolute, feature of the target. This hypothesis is consistent with previous findings that have shown top-down tuning to the target's relative color (Becker, 2010a; Becker et al., 2010, 2013), as well as with the results of Folk and Remington (2008): As they did not vary the target feature value independently of the target-nontarget relationships, it is unclear whether their results were due to target feature certainty or certainty about the target-nontarget relationships.

Context-dependent encoding of features in early, but maybe not late, processes

The present study differs from the majority of other priming studies in that it centrally measured eye movements. Eye movements probe visual search performance at a relatively early point in time - at the stage at which an item is visually selected. Previous studies on feature priming have mostly measured manual RTs (e.g., Hillstrom, 2000; Hodsoll et al., 2006; Huang et al., 2004; Huang \& Pashler, 2005; Kristjánsson \& Driver, 2008; Lamy et al., 2008; Maljkovic \& Nakayama, 1994; Wolfe et al., 2003). RTs probe visual search at a rather late stage of response execution, and therefore include effects on later processes that are concerned, for instance, with response selection (e.g., Becker, 2008a, 2008b, 2008c, 2010a, 2010b).

The present study permits comparing priming effects on eye movements, which index early processes of visual selection, with manual RT, which reflect both early and late processes (see Tables 2, 3, 4 and 5). Inspection of the data reveals that switch costs in the mean RTs largely corresponded to the switch costs found in the proportions and latencies of first target fixations. However, a notable exception was the half-switch condition: Two of the three samerelation conditions (Exps. 1B and 3) showed sizable switch costs in mean RTs that were not reflected in any of the eye movement parameters (i.e., proportions and latencies of first target fixations and mean numbers of nontarget fixations prior to target selection were all unaffected). This partial dissociation between switch costs in early eye movement measures and late RT measures indicates that half-switches can interfere with late processes that commence after the target has been selected. Apparently, processes involved in object identification or response selection can profit from direct repetitions of the target feature value, and/or are slowed by the requirement to process a previously rejected feature (Becker, 2010a, 2010b; Cohen \& Magen, 1999; Mortier, Theeuwes, \& Starreveld, 2005). More importantly, the finding that priming can selectively affect late processes in visual search suggests that mean RTs may not allow safe inferences about the factors and mechanisms that drive visual selection. 


\section{Conclusion}

The present study has yielded several interesting findings about the feature-priming effect: (1) that a shape can be encoded relative to other shapes in the context, (2) that changes of the nontarget shapes alone can impair target selection, despite target feature constancy, and (3) that half-switch conditions do not impair target selection when the target-nontarget relationships remain constant (though they may impair later processes concerned with target identification and/or response selection). Taken together, the results favor a relational account of priming over current feature-based accounts, and show that priming effects are not contingent on target feature certainty, but (if at all) on certainty about the target-nontarget relationships.

Author note This research was supported by ARC Discovery Grant and Postdoctoral Fellowship No. DP110100588, awarded to S.I.B.

\section{References}

Arguin, M., \& Saumier, D. (2000). Conjunction and linear nonseparability effects in visual shape encoding. Vision Research, 40, 3099-3115.

Awh, E., Belopolsky, A. V., \& Theeuwes, J. (2012). Top-down versus bottom-up attentional control: A failed theoretical dichotomy. Trends in Cognitive Sciences, 16, 437-443. doi:10.1016/ j.tics.2012.06.010

Bauer, B., Jolicœur, P., \& Cowan, W. B. (1995). Visual search for colour targets that are or are not linearly separable from distractors. Vision Research, 36, 1439-1465.

Becker, S. I. (2008a). Can intertrial effects of features and dimensions be explained by a single theory? Journal of Experimental Psychology. Human Perception and Performance, 34, 1417-1440. doi:10.1037/a0011386

Becker, S. I. (2008b). The mechanism of priming: Episodic retrieval or priming of pop-out? Acta Psychologica, 127, 324-339. doi:10.1016/j.actpsy.2007.07.005

Becker, S. I. (2008c). The stage of priming: Are intertrial repetition effects attentional or decisional? Vision Research, 48, 664-684. doi:10.1016/j.visres.2007.10.025

Becker, S. I. (2010a). The role of target-distractor relationships in guiding attention and the eyes in visual search. Journal of Experimental Psychology. General, 139, 247-265. doi:10.1037/a0018808

Becker, S. I. (2010b). Testing a postselectional account of acrossdimension switch costs. Psychonomic Bulletin \& Review, 17, 853-861. doi:10.3758/PBR.17.6.853

Becker, S. I. (2013). Guidance of attention by feature relationships: The end of the road for feature map theories? In M. Horsley, M. Eliot, R. Riley, \& B. Knight (Eds.), Current trends in eye tracking research. Berlin, Germany: Springer.

Becker, S. I., Folk, C. L., \& Remington, R. W. (2010). The role of relational information in contingent capture. Journal of Experimental Psychology. Human Perception and Performance, 36, 1460-1476. doi:10.1037/a0020370

Becker, S. I., Folk, C. L., \& Remington, R. W. (2013). Attentional capture does not depend on feature similarity, but on targetnontarget relations. Psychological Science.
Becker, S. I., \& Horstmann, G. (2009). A feature-weighting account of priming in conjunction search. Attention, Perception, \& Psychophysics, 71, 258-272. doi:10.3758/APP.71.2.258

Bichot, N. P., \& Schall, J. D. (2002). Priming in macaque frontal cortex during popout visual search: Feature-based facilitation and location-based inhibition of return. Journal of Neuroscience, 22, 4675-4685.

Brascamp, J. W., Blake, R., \& Kristjánsson, A. (2011). Deciding where to attend: Priming of pop-out drives target selection. Journal of Experimental Psychology. Human Perception and Performance, 37, 1700-1707.

Cohen, A., \& Magen, H. (1999). Intra- and cross-dimensional visual search for single-feature targets. Perception \& Psychophysics, 61, 291-307. doi:10.3758/BF03206889

D’Zmura, M. (1991). Color in visual search. Vision Research, 31, 951-966.

Deubel, H., \& Schneider, W. X. (1996). Saccade target selection and object recognition: Evidence for a common attentional mechanism. Vision Research, 36, 1827-1837. doi:10.1016/00426989(95)00294-4

Eimer, M., Kiss, M., \& Cheung, T. (2010). Priming of pop-out modulates attentional target selection in visual search: Behavioral and electrophysiological evidence. Vision Research, 50, 13531361.

Fecteau, J. H. (2007). Priming of pop-out depends upon the current goals of observers. Journal of Vision, 7(6):1, 1-11. doi:10.1167/7.6.1

Folk, C. L., \& Remington, R. W. (2008). Bottom-up priming of topdown attentional control settings. Visual Cognition, 16, 215-231. doi:10.1080/13506280701458804

Geyer, T., Müller, H. J., \& Krummenacher, J. (2006). Cross-trial priming in visual search for singleton conjunction targets: Role of repeated target and distractor features. Perception \& Psychophysics, 68, 736-749. doi:10.3758/BF03193697

Hillstrom, A. P. (2000). Repetition effects in visual search. Perception \& Psychophysics, 62, 800-817. doi:10.3758/BF03206924

Hodsoll, J. P., Humphreys, G. W., \& Braithwaite, J. J. (2006). Dissociating the effects of similarity, salience, and top-down processes in search for linearly separable size targets. Perception \& Psychophysics, 68, 558-570. doi:10.3758/ BF03208758

Hoffman, J. E., \& Subramaniam, B. (1995). The role of visual attention in saccadic eye movements. Perception \& Psychophysics, 57, 787-795. doi:10.3758/BF03206794

Huang, L., Holcombe, A. O., \& Pashler, H. (2004). Repetition priming in visual search: Episodic retrieval, not feature priming. Memory \& Cognition, 32, 12-20. doi:10.3758/BF03195816

Huang, L., \& Pashler, H. (2005). Expectation and repetition effects in searching for featural singletons in very brief displays. Perception \& Psychophysics, 67, 150-157. doi:10.3758/ BF03195018

Kowler, E., Anderson, E., Dosher, B., \& Blaser, E. (1995). The role of attention in the programming of saccades. Vision Research, 35, 1897-1916. doi:10.1016/0042-6989(94)00279-U

Kristjánsson, Á. (2006). Simultaneous priming along multiple feature dimensions in a visual search task. Vision Research, 46, 2554 2570 .

Kristjánsson, Á., \& Driver, J. (2008). Priming in visual search: Separating the effects of target repetition, distractor repetition and rolereversal. Vision Research, 48, 1217-1232. doi:10.1016/ j.visres.2008.02.007

Kristjánsson, Á., Vuilleumier, P., Schwartz, S., Macaluso, E., \& Driver, J. (2007). Neural basis for priming of pop-out during visual search revealed with fMRI. Cerebral Cortex, 17, 1612-1624. doi:10.1093/cercor/bhl072

Kristjánsson, Á., Wang, D. L., \& Nakayama, K. (2002). The role of priming in conjunctive visual search. Cognition, 85, 37-52. doi:10.1016/S0010-0277(02)00074-4 
Lamy, D., Antebi, C., Aviani, N., \& Carmel, T. (2008). Priming of popout provides reliable measures of target activation and distractor inhibition in selective attention. Vision Research, 48, 30-41.

Leonard, C. J., \& Egeth, H. E. (2008). Attentional guidance in singleton search: An examination of top-down, bottom-up, and intertrial factors. Visual Cognition, 16, 1078-1091.

Maljkovic, V., \& Nakayama, K. (1994). Priming of pop-out: I. Role of features. Memory \& Cognition, 22, 657-672. doi:10.3758/ BF03209251

McPeek, R. M., Maljkovic, V., \& Nakayama, K. (1999). Saccades require focal attention and are facilitated by a short-term memory system. Vision Research, 39, 1555-1566.
Mortier, K., Theeuwes, J., \& Starreveld, P. (2005). Response selection modulates visual search within and across dimensions. Journal of Experimental Psychology. Human Perception and Performance, 31, 542-557.

Wolfe, J. M. (1994). Guided Search 2.0: A revised model of visual search. Psychonomic Bulletin \& Review, 1, 202-238. doi:10.3758/ BF03200774

Wolfe, J. M., Butcher, S. J., Lee, C., \& Hyle, M. (2003). Changing your mind: On the contributions of top-down and bottom-up guidance in visual search for feature singletons. Journal of Experimental Psychology. Human Perception and Performance, 29, 483-502. doi:10.1037/0096-1523.29.2.483 